\title{
Effects of a no-go Task 2 on Task 1 performance in dual - tasking: From benefits to costs
}

\author{
Markus Janczyk $^{1} \cdot$ Lynn Huestegge ${ }^{2}$
}

Published online: 27 December 2016

(C) The Psychonomic Society, Inc. 2016

\begin{abstract}
When two tasks are combined in a dual-task experiment, characteristics of Task 2 can influence Task 1 performance, a phenomenon termed the backward crosstalk effect (BCE). Besides instances depending on the (spatial) compatibility of both responses, a particularly interesting example was introduced by Miller (2006): If Task 2 was a no-go task (i.e., one not requiring any action at all), responses were slowed in Task 1. Subsequent work, however, also reported the opposite result - that is, faster Task 1 responses in cases of no-go Task 2 trials. We report three experiments aiming to more precisely identify the conditions under which a no-go Task 2 facilitates or impedes Task 1 performance. The results suggest that an adverse no-go BCE is only observed when the Task 2 response(s) are sufficiently prepared in advance, yielding strong inhibitory control demands for Task 2 that eventually hamper Task 1 processing as well (i.e., inhibitory costs). If this is not the case, encountering a no-go Task 2 trial facilitates Task 1 performance, suggesting that the underlying task representation is reduced to a single - task. These results are discussed in the context of other recent work on BCEs and of recently suggested accounts of the no-go BCE.
\end{abstract}

Keywords Dual - task · Backward crosstalk · No-go · Preparation · Inhibition

Markus Janczyk

markus.janczyk@uni-tuebingen.de

1 Department of Psychology, Eberhard Karls University of Tübingen, Schleichstraße 4, 72076 Tübingen, Germany

2 Department of Psychology, Julius Maximilians University, Würzburg, Germany
It is a well-known empirical finding that doing two tasks at the same time - thus, dual-tasking - results in performance decrements in at least one of these tasks, in comparison to when the tasks are performed in isolation (Pashler, 1994; for a discussion of possible exceptions, see Janczyk, Pfister, Wallmeier, \& Kunde, 2014). In most dual-task studies, each task is associated with an overt response, so that the conditions involving two responses (i.e., typical dual-task conditions) yield longer response times (RTs) and/or more errors than do conditions involving only one response (i.e., typical singletask conditions). However, there is at least one notable exception: When Task 1 requires an overt response, but Task 2 involves a stimulus signaling not to respond in Task 2 (a nogo condition), Task 1 performance can show performance decrements (as compared with Task 2 go conditions). This adverse no-go backward crosstalk effect (no-go BCE) thus appears to contradict the general rule that two overt actions are harder to process than is only one. In the present study, we set out to examine the underlying factors determining whether a no-go Task 2 yields performance costs versus benefits.

\section{The psychological refractory period and backward crosstalk}

A widely used setup in dual-task research is the psychological refractory period (PRP) paradigm (see Pashler, 1994). On each trial, two stimuli S1 and S2 appear with a varying stimulus onset asynchrony (SOA), and two responses R1 and R2 are required. Although RT1 is mostly unaffected by the SOA variation, RT2 exhibits a large decrease with increasing SOAs, the PRP effect (see Telford, 1931). To explain this effect, Pashler (1994, following Welford 1952) assumed a central bottleneck of response selection: When S2 has been perceptually processed while Task 1 response selection is still 
ongoing, Task 2 response selection must wait until resources from the bottleneck stage are released and available for further processing. However, over the years several findings have raised concerns about the strictly serial and encapsulated response selection view (see also Mittelstädt \& Miller, 2016). In particular, observing that the characteristics of R2 can affect Task 1 performance - hence, the BCE-is hard to reconcile with a basic response selection bottleneck framework.

The first systematic demonstration of a BCE was provided by Hommel (1998). In his experiments, the participants were presented colored letters as stimuli and - in most experimentsgave two subsequent responses: R1 to the color, and R2 to the identity. If both responses were "left" or both were "right" (i.e., R1-R2 compatible), even the RTs in Task 1 were faster than those from trials in which one response was "left" but the other "right" (R1-R2 incompatible). Similar results were subsequently reported by various other researchers, and hence this kind of a BCE appears to be rather stable across different tasks, stimuli, and responses (e.g., Ellenbogen \& Meiran, 2008; Hommel \& Eglau, 2002; Janczyk, 2016; Janczyk, Büschelberger, \& Herbort, 2016; Janczyk, Pfister, Hommel, \& Kunde, 2014; Lien \& Proctor, 2000). Typically, this BCE is explained via the assumption of automatic response activation occurring in Task 2 that feeds into either response activation (Hommel, 1998; Lien \& Proctor, 2002) or response selection (Janczyk, Renas, \& Durst, 2016; Thomson, Danis, \& Watter, 2015) of Task 1.

Another particularly interesting type of BCEs was introduced by Miller (2006). In this PRP study, Task 1 was a standard choice reaction task, but Task 2 was a go/no-go task (with only one possible go response), and even in this case (i.e., without any need to respond in Task 2), Task 2 processing affected Task 1 performance. However, although one would perhaps expect faster RT1s in the case of no-go Task 2 trials (because only one response must be produced in this case), the opposite was true: RT1s were slower in Task 2 nogo trials (an adverse no-go BCE; see also Ko \& Miller, 2014). One interpretation is that in the case of no-go trials a prepared response must be inhibited, and this inhibition spreads to Task 1 , resulting in slower R1s as well (for a different interpretation, in terms of "abstract compatibility," see Röttger \& Haider, 2016, and the General Discussion). Thus, these Task 2 no-go conditions are apparently not represented in the same way as a single-task (Task 1 only) condition, but additional inhibitory demands regarding Task 2 appear to be involved, hampering Task 1 performance. However, the no-go BCE seems to be less stable than the R1-R2 BCE across the various specific paradigms used to assess BCEs.

\section{A closer look at the no-go BCE}

Another set of studies in which a no-go BCE was assessed used a variant of the PRP paradigm, known as the prioritized- processing (PP) paradigm (Miller \& Durst, 2014, 2015). In this variant, both Task 1 and Task 2 are choice/no-go tasks (i.e., two go responses are possible). Importantly, Task 2 is only to be processed in Task 1 no-go trials. Thus, on each trial, only one response is required, and clear priority is given to Task 1, since S2 need not even be considered if Task 1 is a go trial. In many respects, the performance patterns in the PP paradigm are reconcilable with the bottleneck model used to explain the PRP effect (see, in particular, Miller \& Durst, 2015).

In Experiment 1 of Miller and Durst (2014), participants gave both responses with either the left or the right index finger, and the fastest RT1s were observed in R1-R2 compatible trials, intermediate RT1s in R1-R2 incompatible trials, and the slowest RT1s if Task 2 was a no-go trial (even though none of these possible R2s actually had to be carried out). ${ }^{1}$ The results revealed both an R1-R2 and a no-go BCE in this experiment, and similarly so in Experiments 1 and 2 of Miller and Durst (2015). In contrast, using different sets of effectors for both tasks, no R1-R2 BCE was reported in Miller and Durst's (2014) Experiment 2. At the same time, a no-go BCE was evident for RT1s, but it was compromised by a speed-accuracy trade-off. Thus, one should rather conclude that no type of BCE was clearly present in this experiment, and the same pattern was reported in Experiments 3 and 4 of Miller and Durst (2015). In contrast, when using a standard PRP paradigm, Miller and Durst (2015) observed even faster RT1s when Task 2 was a no-go trial (Exp. 3), suggesting that participants represented the corresponding task demands as a single Task 1 (i.e., without any additional inhibitory demands affecting Task 1 processing). For the sake of completeness, recent work by Mittelstädt and Miller (2016) reported an R1R2 BCE in a PRP setup, but not in a PP setup, when compatible responses were given with different fingers but the same hand. In sum, in these previous experiments an adverse no-go $\mathrm{BCE}$ was either present, absent, or reversed, suggesting different mental representations of the task demands.

\section{The present experiments}

With the present experiments, we aimed to present a systematic investigation to further pinpoint under which conditions a no-go S2 facilitates or impedes Task 1 performance. Across experiments, we addressed two features of the experimental design that might be relevant-namely, the separation of the stimulus information, and the ease of Task 2 advance response preparation. In previous no-go BCE studies, the stimuli for both tasks were usually spatially and temporally separated, which might have affected the availability and saliency (and

\footnotetext{
${ }^{1}$ Note that in these works, the RT1 in a PP experiment is generally denoted the primary-task $\mathrm{RT}\left(\mathrm{RT}_{\mathrm{P}}\right)$, and $\mathrm{RT} 2$ the background-task $\mathrm{RT}\left(\mathrm{RT}_{\mathrm{B}}\right)$.
} 
thus, the processing) of, for example, the Task 2 no-go signal. When stimuli are temporally separated, the no-go information for Task 2 is not available at the beginning of a trial. Thus, it is more reasonable to remain prepared to execute Task 2 in these cases (higher probability of Task 2 advance preparation) than in conditions in which participants already know at the beginning of a trial that a Task 2 response will not be required. Here, we thus explored a situation in which all relevant information for both tasks was presented at the same time (the beginning of a trial) by utilizing a single (multidimensional) stimulus. In addition, we varied whether the Task 2 go/no-go information was represented in the same dimension of the stimulus as the choice information versus in a different dimension. When the no-go information is available earlier, it might be easier for participants to represent the corresponding task demands in terms of a single - task (predicting a beneficial no-go BCE). We further varied the ease of advance response preparation by using either a choice/no-go task (two overt response alternatives) or a simple go/no-go (one overt response alternative) task, reasoning that the latter should be easier to prepare (and thus more likely to be prepared) in advance (therefore yielding higher inhibition costs; i.e., an adverse no-go BCE).

Specifically, in Experiment 1, an R1-R2 and a no-go BCE paradigm were implemented jointly, but the response location information and the go/no-go information of Task 2 were distributed across two dimensions of the stimuli. In Experiments 2 and 3, the whole paradigm was made more similar to the Miller (2006) paradigm, while at the same time replicating the results of the previous experiments within the same sample of participants. To anticipate the main results of the present study, in Experiments 1, 2, and 3a, Task 1 performance was much improved in the case of Task 2 no-go trials. Only in Experiment 3b, when Task 2 was a simple go/no-go task with only one possible go response - which could thus be entirely prepared — did Task 1 performance suffer during Task 2 no-go trials.

\section{Experiment 1}

Here, we combined the experimental paradigms used to study the R1-R2 BCE (Hommel, 1998; Janczyk, 2016) and the nogo BCE (Miller, 2006) within one experiment. Thus, the required responses in Task 1 and Task 2 could be spatially compatible, but at the same time Task 2 also entailed no-go trials. Deviating from the experiments of Miller and Durst (2014, 2015), however, the response location information and go/ no-go information of Task 2 were distributed across two dimensions of the same single stimuli, and thus all relevant information for both tasks was present at the beginning of each trial. Specifically, the size of a geometrical shape determined a left or right manual R1, its color determined a left or right pedal R2 in go trials, and the particular shape determined whether R2 was to be given (go trial) or not given (no-go trial).

\section{Method}

Participants A group of 24 undergraduate students from the University of Tübingen participated (20 female, four male; mean age $=20.3$ years). The participants signed informed consent prior to the experiment and were naïve with regard to the underlying hypotheses.

Apparatus and stimuli Response collection and stimulus presentation were done with a standard PC connected to a 17-in. CRT monitor. Manual responses were collected with two external, custom-built response keys placed to the left and right of the participant. Pedal responses were collected with two foot pedals placed on the floor. The visual stimuli were a rectangle and a diamond (shape) that were either small or large (size) and either green or red (color), presented on a black background.

Task and procedure On each trial, participants performed two tasks, Tasks 1 and 2, respectively. The Task 1 response (R1) was to be given on each trial via a left/right keypress with the left/right index finger. The Task 2 response (R2) was given with the left/right foot on the respective foot pedal; however, this R2 was only to be executed on $50 \%$ of the trials (go trials). On the other $50 \%$ of the trials, R2 must not be executed (no-go trials). The size of the stimuli determined the manual R1, its color determined the pedal R2, and the shape determined whether R2 was to be given (go trial) or not given (no-go trial).

A trial started with a central white fixation cross $(250 \mathrm{~ms})$, followed by a blank screen $(250 \mathrm{~ms})$ and the onset of the central visual stimulus. Both responses were to be given serially, in the case of a go trial (i.e., manual prior to foot response), and a trial ended after 4,000 $\mathrm{ms}$ if not all required responses were executed prior to this deadline. Specific error feedback was given $(1,000 \mathrm{~ms})$ in the cases of wrong responses or general errors ( $\mathrm{R} 2$ given in no-go trials, $\mathrm{R} 2$ given before R1, etc.). Participants first performed 20 randomly drawn familiarization trials, followed by 12 blocks of 48 trials resulting from six repetitions of 2 Shapes $\times 2$ Colors $\times 2$ Sizes. The first two blocks were considered practice and were not analyzed. Written instructions emphasized both speed and accuracy. Participants were tested individually in a single 50min session, and the assignments of stimulus dimension levels to the required responses were counterbalanced across participants.

Design and analyses Trials were classified as to whether (1) both R1 and R2 were to given on the same (R1-R2 
compatible) or different sides (R1-R2 incompatible) and (2) R2 was actually to be executed (go trial) or not (no-go trial). For the RT analyses, only correct trials were considered, and RTs were deemed outliers when they deviated from the mean by more than $2.5 S D$ s (calculated separately for each participant and design cell). ${ }^{2}$

Our analyses focused on RTs and percentage errors (PEs). Both variables were submitted to analyses of variance (ANOVA) with R1-R2 compatibility and go/no-go as repeated measures variables.

\section{Results}

Errors in response to the go/no-go feature (i.e., responses in no-go trials, or no responses in go trials) were rare (between zero and nine per participant; i.e., $0-1.9 \%$ of trials).

Task 1 The mean correct RT1s (2.89\% outliers) are visualized in Fig. 1 (see also Table 1). They were overall $29 \mathrm{~ms}$ faster in R1-R2 compatible (vs. incompatible) trials, $F(1,23)=9.57, p$ $=.005, \eta_{\mathrm{p}}{ }^{2}=.29$. Importantly, RT1s were $101 \mathrm{~ms}$ faster in nogo than in go trials, $F(1,23)=36.21, p<.001, \eta_{\mathrm{p}}{ }^{2}=.61$. We observed a trend toward a smaller R1-R2 BCE in no-go trials, but the interaction was not significant, $F(1,23)=2.93, p=$ $.101, \eta_{\mathrm{p}}^{2}=.11$.

PE1s are also summarized in Table 1. In general, participants made very few errors. Statistically, more errors occurred in R1-R2 compatible (vs. incompatible) trials, $F(1,23)=$ $17.52, p<.001, \eta_{\mathrm{p}}{ }^{2}=.43$. We observed no overall difference based on the go versus no-go status of Task $2, F(1,23)=1.09$, $p=.308, \eta_{\mathrm{p}}{ }^{2}=.05$. Numerically, for $\mathrm{R} 1-\mathrm{R} 2$ compatible trials more errors were made on go trials, but this was reversed for no-go trials, yielding a significant interaction, $F(1,23)=$ $14.66, p=.001, \eta_{\mathrm{p}}{ }^{2}=.39$.

Task 2 The mean correct RT2s (2.46\% outliers; only go trials) were 1,193 and 1,121 ms for R1-R2 incompatible and compatible trials, respectively, $F(1,23)=14.11, p=.001, \eta_{\mathrm{p}}{ }^{2}=$ .38. Response errors in go trials occurred in 0.8 and $2.7 \%$ of the incompatible and compatible trials, $F(1,23)=0.01, p=$ $.916, \eta_{\mathrm{p}}^{2}<.01$.

\section{Discussion}

In Experiment 1, we combined an R1-R2 compatibility-based BCE (Hommel, 1998) with a Task 2 go/no-go-based BCE

\footnotetext{
${ }^{2}$ A problem with interpreting R1-R2 BCEs is response grouping - that is, waiting until both responses (in go trials) have been selected, and then emitting them in rapid succession. We thus reran all analyses reported in this article while excluding go trials in which the two responses were not separated by at least $100 \mathrm{~ms}$. This yielded only minor changes in statistics, but the general qualitative pattern remained the same. Thus, the results are reported on the basis of all trials.
}

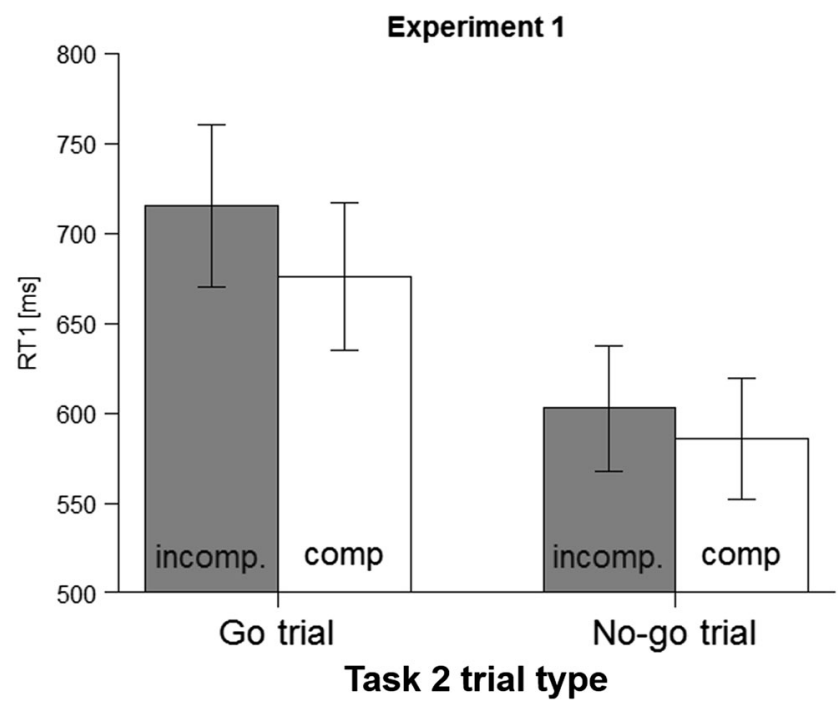

Fig. 1 Response times [in milliseconds] from Task 1 (RT1s) of Experiment 1 as a function of R1-R2 compatibility and go versus nogo trials on Task 2. Error bars show \pm 1 standard error of the mean

(Miller, 2006). The most interesting result of Experiment 1 was that RT1s were much faster (about $100 \mathrm{~ms}$ ) when Task 2 was a no-go trial (beneficial no-go BCE). This contrasts with the observations of Miller (2006; see also Ko \& Miller, 2014; Miller \& Durst, 2014; Miller \& Durst, 2015, Exps. 1 and 2), who reported slower Task 1 responses when Task 2 was a no-go trial (adverse no-go BCE). A crucial difference from the studies of Miller (2006) and Ko and Miller (2014) is that in their experiments, a separate stimulus for Task 2 was used (which occurred after the Task 1 stimulus), and the Task 2 stimulus was informative only regarding go versus no-go status (i.e., only one go response was possible). In our experiment, though, additional information was provided about which response was required in the case of a go trial, even though both features were signaled by different stimulus dimensions.

Additionally, a typical R1-R2 BCE was observed for RTs (but was undermined by a slight speed-accuracy trade-off), which was numerically smaller for no-go than for go trials. It is tempting to interpret this trend toward an interaction in terms of Sternberg's additive-factor logic (Sternberg, 1969): A statistical interaction of two manipulations would indicate that both manipulations affected similar stages of processing, whereas an additive combination would indicate that they affected different stages. In the present context, however, such an interpretation is difficult. One reason is the speed-accuracy trade-off in the present data, and another is that the R1-R2 BCE increased with the overall RT level (Hommel, 1998) - and the RTs were clearly different for the go and the no-go trials (i.e., RT1s were about $100 \mathrm{~ms}$ faster in no-go than in go trials). Finally, it is perhaps not surprising that the R1-R2 BCE was much smaller without an overt response in Task 2 (cf. Hommel, 1998, Exp. 5; Janczyk, Renas et al., 2016, Exp. 2), since there was less need to represent $\mathrm{R} 2$ features in the first place. 
Table 1 Response times and percentage errors from Task 1 of Experiment 1 as a function of R1-R2 compatibility and go versus no-go trials

\begin{tabular}{lllll}
\hline & \multicolumn{2}{l}{ Response Time $[\mathrm{ms}]$} & & \multicolumn{2}{c}{ Percentage Error } \\
\cline { 2 - 3 } & Go Trials & No-Go Trials & & Go Trials \\
\hline R1-R2 compatible & 675 & 586 & 2.7 & 1.7 \\
R1-R2 incompatible & 715 & 603 & 0.8 & 1.1 \\
BCE & 40 & 17 & -1.9 & -0.6 \\
\hline
\end{tabular}

The backward crosstalk effect (BCE) was calculated by subtracting the value from the R1-R2 compatible conditions from that of the incompatible condition.

In any case, before drawing further conclusions from the present results, in Experiment 2 we aimed to replicate and extend them.

\section{Experiment 2a and 2b}

In light of Miller's (2006) results, the faster RT1s in the no-go trials of Experiment 1 were somewhat unexpected. One possible interpretation would be that participants first attended the go/no-go feature only to evaluate the go/no-go status, and in the case of no-go trials, "reduced" the dual-task to a singletask representation. However, some design differences were also apparent. The major differences were (1) that the stimulus also suggested a choice task (left vs. right response) in the case of go trials (instead of utilizing only a single possible go response) and (2) that the information about the correct response and the go/no-go status were distributed across two different stimulus dimensions (color and shape) versus a single one.

Experiment 2 had two goals: First, we aimed to replicate the reversed (i.e., beneficial) no-go BCE observed in Experiment 1 with a new sample of participants (Exp. 2a). Second, the same group also performed a similar experiment (Exp. 2b), but now the Task 2 go/no-go status and the correct response in the case of go trials were defined by one stimulus dimension (color), to discourage participants from first attending only the go/no-go feature.

\section{Method}

Participants A group of 24 new undergraduate students from the University of Tübingen participated ( 20 female, four male; mean age $=23.3$ years) and fulfilled the same criteria as in Experiment 1. Each participant took part in both Experiments $2 \mathrm{a}$ and $2 \mathrm{~b}$.

Apparatus, stimuli, task, and procedure In many respects the experiments resembled Experiment 1, and here only the changes are described. Experiment $2 a$ differed from Experiment 1 in that only six blocks were administered. In Experiment $2 b$, yellow was introduced as a third color. Task 1 was again to respond to the size with a manual left/right keypress. Two of the colors were assigned a left/right pedal go response, and the third color was assigned a no-go response. Although it was irrelevant to the present task, stimulus shape still varied, to maximize the similarity between experiments. Each block comprised 48 trials, resulting from four repetitions of 2 Shapes $\times 3$ Colors $\times 2$ Sizes. The first blocks were considered practice and not analyzed. The stimulus-response mappings were randomly drawn at the beginning of the experiments, and the same "S1-size to R1" mapping was used for both experiments. The order of the two experiments was counterbalanced across participants.

Design and analyses The analyses in Experiment 2a were the same as those in Experiment 1 . The data of Experiment $2 b$ were analyzed as a function of trial type, with the levels R1-R2 compatible go trial, R1-R2 incompatible go trial, and no-go trial (i.e., there was no variation of compatibility in the case of no-go trials).

\section{Results}

Experiment 2a Errors in terms of the go/no-go feature (i.e., responses in no-go trials, or no responses in go trials) were rare (between zero and six per participant; i.e., $0-2.7 \%$ of trials).

The mean correct RT1s (2.81\% outliers) are visualized in Fig. 2 (left panel; see also Table 2). By and large, the results replicated those of Experiment 1. First, we observed an overall R1-R2 compatibility effect, and RT1s were overall $34 \mathrm{~ms}$ faster in R1-R2 compatible trials, $F(1,23)=5.29, p=.031$, $\eta_{\mathrm{p}}{ }^{2}=.19$. Second, RT1s were about $130 \mathrm{~ms}$ faster in no-go than in go trials, $F(1,23)=20.44, p<.001, \eta_{\mathrm{p}}{ }^{2}=.47$. Third, we also observed a trend toward a smaller R1-R2 BCE in nogo trials, but the interaction again just missed conventional significance, $F(1,23)=3.86, p=.062, \eta_{\mathrm{p}}{ }^{2}=.14$.

PE1s are also summarized in Table 2. Participants again made more errors in R1-R2 compatible trials, $F(1,23)=$ $4.67, p=.041, \eta_{\mathrm{p}}{ }^{2}=.17$. All other effects did not reach conventional significance, all $F_{\mathrm{s}} \leq 3.71$, all $p \mathrm{~s} \geq .067$.

The mean correct RT2s ( $2.42 \%$ outliers; only go trials) were 1,318 and 1,257 ms for R1-R2 incompatible and compatible trials, respectively, $F(1,23)=6.27, p=.020, \eta_{\mathrm{p}}{ }^{2}=.21$. Response errors on go trials occurred in 1.5 and $2.5 \%$ of the 


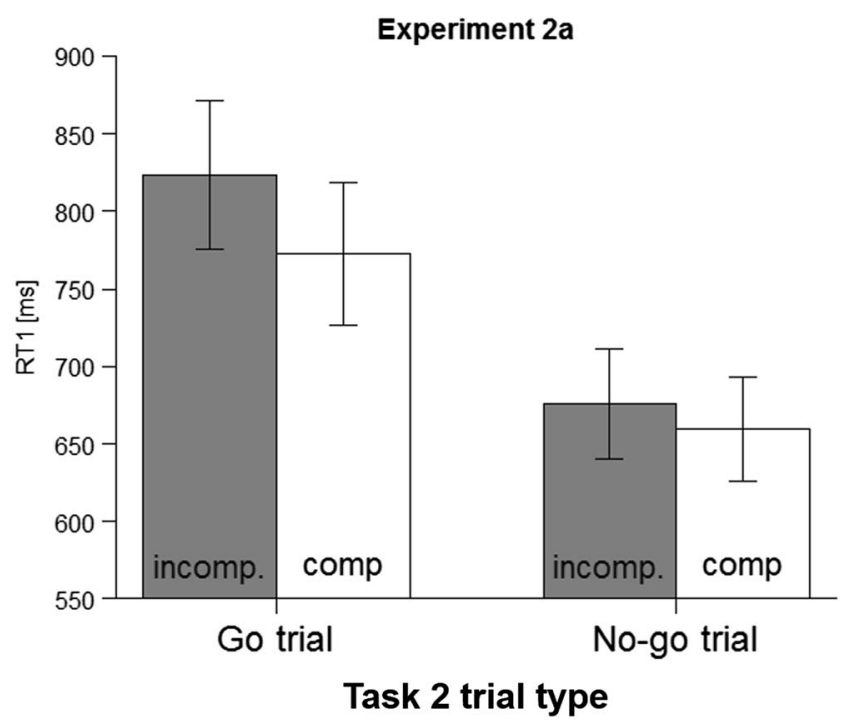

Fig. 2 (Left) Response times [in milliseconds] from Task 1 (RT1s) of Experiment $2 \mathrm{a}$ as a function of R1-R2 compatibility and go versus no-go trials. (Right) RT1s of Experiment $2 \mathrm{~b}$ as a function of Task 2 trial type.

incompatible and the compatible trials, respectively, $F(1,23)$ $=0.12, p=.729, \eta_{\mathrm{p}}^{2}=.01$.

Experiment 2b In general, errors in response to the go/no-go feature (i.e., responses in no-go trials, or no responses in go trials) were rare (between zero and nine per participant; i.e., 0 $3.8 \%$ of the trials). However, one exceptional participant made 79 such errors (33.2\% of the trials). The qualitative pattern of results did not change when these data were excluded, so we decided to report the results on the basis of all participants.

The mean correct RT1s (2.65\% outliers) are visualized in Fig. 2 (right panel). The main effect of trial type was significant, $F(2,46)=13.29, p<.001, \eta_{\mathrm{p}}{ }^{2}=.37, \varepsilon=.74$, with the slowest responses in R1-R2 incompatible trials $(710 \mathrm{~ms})$, intermediate RT1s in R1-R2 compatible trials ( $679 \mathrm{~ms})$, and the fastest RT1s in Task 2 no-go trials (619 ms). Participants made more errors in R1-R2 incompatible (1.9\%) than in R1-R2 compatible go trials $(1.1 \%)$ and in no-go trials $(1.1 \%)$, and the main effect of trial type was significant, $F(2,46)=3.84, p=.029, \eta_{\mathrm{p}}{ }^{2}=.14$.

The mean correct RT2s (2.72\% outliers; go trials only) were 1,147 and 1,1107 ms in R1-R2 incompatible and compatible trials, respectively, $F(1,23)=7.45, p=.012, \eta_{\mathrm{p}}^{2}=$

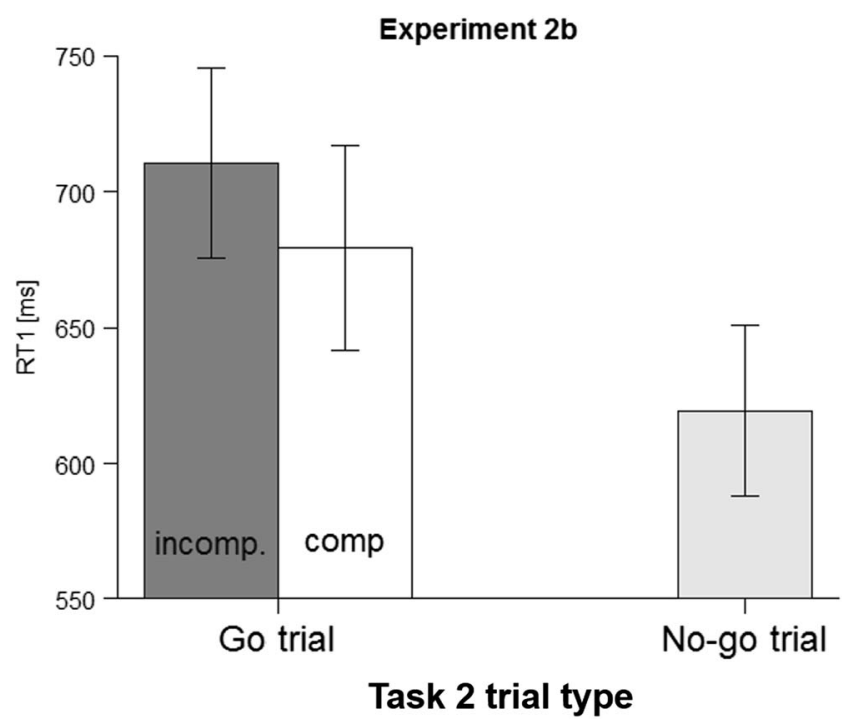

Error bars show \pm 1 standard error of the mean. incomp., R1-R2 incompatible go trial; comp., R1-R2 compatible go trial

.245. The mean PE2s were 3.5 and $3.2 \%, F(1,23)=0.09, p$ $=.761, \eta_{\mathrm{p}}^{2}<.01$.

\section{Discussion}

The purpose of Experiment 2 was twofold. As a first purpose, Experiment 2a was run to test whether we could replicate the results obtained in Experiment 1, and indeed, they were largely replicated. Again, we observed a main effect of R1-R2 compatibility, but - most importantly-also faster RT1s in no-go (vs. go) trials - thus, again the opposite of what Miller (2006) reported. Note that again the beneficial no-go BCE was substantial (about $130 \mathrm{~ms}$; i.e., about four times the R1-R2 $\mathrm{BCE}$ ). The interaction approached significance, but is difficult to interpret for the same reasons mentioned in the Discussion of Experiment 1.

One interpretation may be that the participants in Experiment $2 \mathrm{a}$ first attended to the particular stimulus dimension that indicated the go/no-go status, and in no-go trials "reduced" the cognitive demand to a single - task, thereby being able to respond faster to Task 1. Therefore-and this was the second purpose - in Experiment $2 b$ the go/no-go information

Table 2 Response times and percentages errors from Task 1 of Experiment 2a as a function of R1-R2 compatibility and go versus no-go trials

\begin{tabular}{|c|c|c|c|c|}
\hline & \multicolumn{2}{|c|}{ Response Time [ms] } & \multicolumn{2}{|c|}{ Percentage Error } \\
\hline & Go Trials & No-Go Trials & Go Trials & No-Go Trials \\
\hline R1-R2 compatible & 772 & 660 & 2.5 & 1.2 \\
\hline $\mathrm{R} 1-\mathrm{R} 2$ incompatible & 823 & 676 & 1.5 & 1.0 \\
\hline $\mathrm{BCE}$ & 51 & 16 & -1.0 & -0.2 \\
\hline
\end{tabular}

The backward crosstalk effect (BCE) was calculated by subtracting the value from the R1-R2 compatible conditions from that of the incompatible condition. 
was provided within the same stimulus dimension (color) as the left/right R2 information for go trials (see Miller \& Durst, 2014). In the case of go trials, a standard R1-R2 BCE resulted. However, Task 1 responses again were much faster in the case of Task 2 no-go trials. Thus, we can rule out that coding the go/ no-go information on a separate (vs. the same) stimulus dimension is a relevant factor that determines adverse versus beneficial no-go BCEs. Still, design differences existed in comparison to Miller's (2006) experiments, in which only one response was possible in the case of go trials, whereas in our Experiment $2 b$ participants needed to decide between a left and a right response. Whether this was critical would be examined in Experiment 3.

\section{Experiments 3a and 3b}

In the previous Experiment $2 \mathrm{~b}$, the no-go information was provided by the same stimulus dimension as the left/right go information (as in Miller \& Durst, 2014). However, we still did not observe an adverse no-go BCE. Experiment 3a therefore replicated our previous procedure, and the same participants performed in Experiment 3b, which was very similar to the go/no-go task used in the experiments of Miller (2006): Here, a simple go/no-go task with only one overt response alternative (instead of two) was employed, to increase the likelihood of advance Task 2 (response) preparation and thus to pose higher demands on inhibitory control in no-go trials.

\section{Method}

Participants A group of 24 new undergraduate students from the University of Tübingen participated (17 female, seven male; mean age $=22.6$ years ) and fulfilled the same criteria as in Experiments 1 and 2. All participants performed in both Experiments $3 \mathrm{a}$ and $3 \mathrm{~b}$.

Apparatus, stimuli, task, and procedure Experiment $3 a$ was the same as Experiment 2b, for direct replication purposes. In Experiment 3b, participants responded with a left/right manual response to the size of the geometrical objects as well (Task 1). Since we reasoned that prelearned associations between the color and go/no-go information (e.g., go $\rightarrow$ green, no-go $\rightarrow$ red) might potentially play a minor role, the color of the objects was now irrelevant in Experiment 3b, but the type of the object determined whether a Task 2 go response was required (go) or not required (no-go). In the case of a go response, participants pressed a foot pedal with their right foot. Note that in this experiment, no left/right decision was to be made anymore in Task 2 .

Design and analyses The analyses in Experiment $3 \mathrm{a}$ were similar to those in Experiment $2 \mathrm{~b}$. The data from Experiment $3 \mathrm{~b}$ were analyzed with one-tailed $t$ tests, and on the basis of the similarity of Experiment $3 \mathrm{~b}$ with the research design of Miller (2006), we here expected to find faster RT1s in Task 2 go trials than in Task 2 no-go trials.

\section{Results}

Experiment 3a In general, errors in response to the go/no-go feature (i.e., responses in no-go trials, or no responses in go trials) were rare (between zero and three per participant; i.e., $0-1.3 \%$ of the trials).

The mean correct RT1s (2.51\% outliers) are visualized in Fig. 3 (left panel). Again, RT1s were slowest in R1-R2 incompatible trials (754 ms), intermediate in R1-R2 compatible trials (739 ms), and fastest in Task 2 no-go trials $(680 \mathrm{~ms}), F(2$,
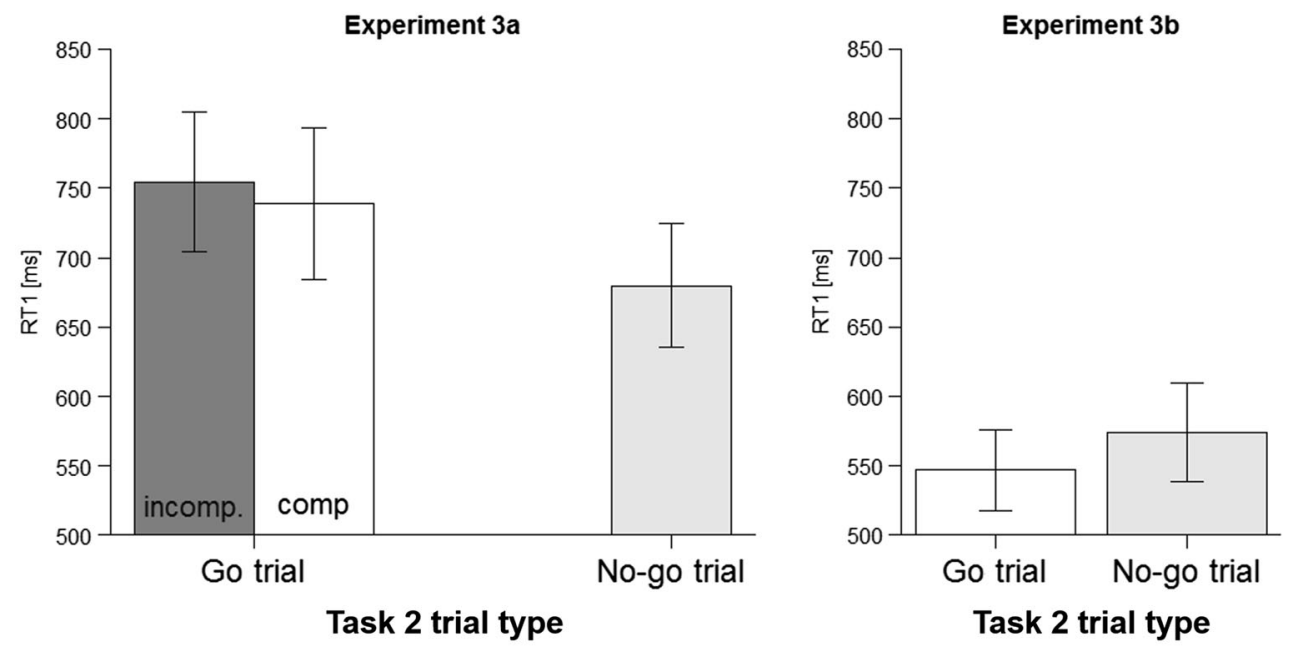

Fig. 3 (Left) Response times [in milliseconds] from Task 1 (RT1s) of Experiment 3a as a function of Task 2 trial type. (Right) RT1s of Experiment 3b as a function Task 2 trial type. Error bars show \pm 1 standard error of the mean. incomp., R1-R2 incompatible go trial; comp., R1-R2 compatible go trial 
46) $=7.29, p=.002, \eta_{\mathrm{p}}{ }^{2}=.24$. The mean PE1s were 2.6, 2.5, and $1.6 \%$ for the incompatible, compatible, and no-go trials, $F(2,46)=1.41, p=.255, \eta_{\mathrm{p}}{ }^{2}=.06$.

The mean correct RT2s ( $2.50 \%$ outliers; go trials only) were 1,184 and 1,156 ms in R1-R2 incompatible and compatible trials, respectively, $F(1,23)=1.33, p=.260, \eta_{\mathrm{p}}{ }^{2}=.05$. The mean PE2s were 3.7 and $4.8 \%$ for incompatible and compatible go trials, $F(1,23)=0.83, p=.371, \eta_{\mathrm{p}}{ }^{2}=.02$.

Experiment 3b Here, for the first time in this study, RT1s were faster when Task 2 required a go response $(547 \mathrm{~ms})$ than when Task 2 required a no-go response $(574 \mathrm{~ms}), t(23)=2.00$, $p=.029$. No differences between Task 2 responses were observed for PEs in Task 1 (no-go trial, 1.7\% vs. go trial, 2.1\%), $t(23)=0.68, p=.747$.

\section{Discussion}

First, the results of Experiment 3 a replicated those of Experiment 2b. In particular, we again observed faster RT1s when Task 2 required a no-go response (beneficial no-go $\mathrm{BCE}$ ). Although the left/right go information was again provided within the same stimulus dimension as the go/no-go information for Task 2, this was changed in Experiment 3b. In this experiment, the same participants performed a task in which the go/no-go information was provided in a single dimension and no left/right go discrimination was required. In this case, we indeed observed longer RT1s when Task 2 required a no-go response (adverse no-go BCE, as in Miller, 2006). At first sight, one might argue this effect was comparatively small. However, it is crucial to compare this adverse no-go BCE effect to the quite substantial beneficial no-go BCE effects observed in the previous experiments. This comparison clearly suggests that a qualitative difference in mental task representations must have taken place, which should be related to the fact that in Experiment 3b, Task 2 go response processing (and thus the potential for advance Task 2 preparation) was made much easier by deleting the additional go response alternative. Furthermore, note that in Miller's (2006) study, both responses were given with hands. In our experiment, the go/no-go tasks involved a foot response. Inhibition applied to such foot responses might spread over to concurrent hand responses less than inhibition of other hand responses would.

\section{General discussion}

In the present study, we set out to investigate the conditions under which a no-go S2 facilitates or impedes Task 1 performance in a dual-task setting. Miller (2006) reported an adverse no-go BCE - that is, longer RT1s when S2 signaled a no-go trial in comparison to a go trial (see also Ko \& Miller, 2014). Subsequent studies suggested a more ambiguous picture
(Miller \& Durst, 2014, 2015), however, by sometimes even reporting a beneficial no-go $\mathrm{BCE}$. In the present study, we utilized a single multidimensional stimulus to provide all relevant information for both tasks (including the go/no-go information) at the beginning of each trial. In all experiments, Task 1 required a left/right manual keypress response based on the size of the stimulus. In Experiments 1 and $2 \mathrm{a}$, the specific location of the left/right Task 2 pedal response and whether it was a go- or a no-go trial was signaled by the color and the shape of the stimulus, respectively; in Experiments $2 \mathrm{~b}$ and $3 \mathrm{a}$, this information was presented within a single (color) stimulus dimension; and in Experiment 3b, the choice/no-go Task 2 was reduced to a simple go (vs. no-go) task (i.e., there was only one overt response alternative).

\section{R1-R2 and no-go BCEs in a dual - task}

Two aspects were present in the results from all experiments except Experiment 3b: The responses in Task 1 were faster when Task 2 required (or at least signaled) a response on the same (vs. the different) side. These findings represent an instance of an R1-R2 BCE (see Hommel, 1998; Janczyk, 2016; and many others). The second, and more interesting, result is that of a beneficial no-go BCE-that is, faster Task 1 responses when Task 2 was a no-go in comparison to a go trial (see also Miller \& Durst, 2015, Exp. 3). Only in Experiment $3 \mathrm{~b}$ was an adverse no-go BCE present. This was the result reported in the original study by Miller (2006), and thus generalizes to the use of pedal responses in Task 2, as well.

What distinguishes Experiment $3 \mathrm{~b}$ from the other experiments is that there was only one possible overt response in Task 2. On go trials in all other experiments, the particular response location (left vs. right) was unknown to the participants in advance of a trial. Miller and Durst (2015) suggested that advance task preparation might be reduced in these cases of a choice/no-go decision, and thus weaker inhibitory activation would arise from Task 2 processing in the case of a no-go trial. Only when full preparation of one response is possible (such as in Miller, 2006) does this prepared response need relatively stronger inhibition in no-go trials, which in turn might affect Task 1 responses as well. Such an explanation seems applicable to our experiments (Exp. 3b) as well, as will be outlined below.

Specifically, we reasoned that finding adverse versus beneficial no-go BCEs depends on the underlying preparatory mental representations of the task demands: When participants represent the task demands in terms of a (more integrated) dual - task as a default representation, deleting one of the tasks (per no-go signal) results in additional inhibitory demands (task inhibition within the default task scheme), leading to the costs associated with inhibition. When participants represent the task demands more distinctly for each component task (default representation in terms of the single - tasks), go trials are similar to dual-task trials, and no-go trials 
correspond to single-task trials, yielding performance costs for go trials. The relevant factor (as reasoned by Miller \& Durst, 2015) appears to be advance task preparation (for Task 2): When advance task preparation is made more likely by making Task 2 easier to prepare, as in simple go/no-go (vs. choice/ no-go) tasks or by withholding the go/no-go information at the beginning of a trial (through the introduction of a lateroccurring stimulus coding the Task 2 information), more inhibitory control is needed to inhibit the response, eventually affecting RT1 (i.e., inhibitory costs). On the other hand, when preparation for Task 2 is made less likely (through a more complex Task 2 or providing no-go information early in the trial), there is nothing to inhibit in the first place, since the task representations resemble those in standard single-task trials.

Note that Experiments 1 and 2 of Miller and Durst (2015) and Experiment 1 of Miller and Durst (2014) showed an adverse no-go BCE, even though they used a choice/no-go task in the PP paradigm. However, these experiments represent a special case, since the Task 1 and Task 2 responses were given with the same fingers. We suggest that the advance preparation in this case related to the effectors involved: Although it was not clear which location must be responded to, it was entirely clear that the response would be given with the same hand as in the other task. When different responses were instead used for both tasks, no adverse no-go BCE was reported.

The present results also speak to a recent study by Röttger and Haider (2016). These authors argued that the (adverse) nogo BCE (Miller, 2006) may also be explained in a similar way as is the R1-R2 BCE: The S2 unambiguously indicates a no-go response, which is - on a more abstract level—incompatible with the go representation of Task 1. Röttger and Haider tested this hypothesis mainly by using free-choice tasks (Berlyne, 1957; Janczyk, Nolden, \& Jolicœur, 2015) in which their participants freely chose whether or not to respond. In this case, no adverse no-go BCE was observed when no-go was (freely) chosen, whereas it was observed when an S2 indicated go (vs. no go). The authors concluded that whenever an $\mathrm{S} 2$ unambiguously indicated a no-go trial, this would automatically activate a no-go representation, negatively affecting Task 1 performance. If this were true, however, the use of a choice/no-go task should yield an adverse no-go BCE: In all our experiments, a particular S2 unambiguously indicated no-go trials, but apparently only in Experiment $3 b$ was an adverse no-go BCE observed. These results are thus difficult to reconcile with the hypothesis proposed by Röttger and Haider (at least without adding additional assumptions that would explain why sometimes the no-go S2 did not automatically activate no-go representations, which in turn would negatively affect Task 1 processing).

One possible counterargument against our interpretation of the results outlined above is that Experiment $3 \mathrm{~b}$ differed in task difficulty from the other experiments, yielding much faster Task 1 RTs. It thus might be the case that no-go representations were in fact activated in all experiments but decayed too fast to affect Task 1 performance in the (more difficult) Experiments 1, 2, and 3a. Although this possibility cannot be ruled out, three arguments speak against the objection. First, this alternative explanation would only explain the absence of adverse BCEs in Experiments 1, 2, and 3a, but not the substantial no-go benefit. Second, in the original Miller (2006) study, the no-go BCE was present across the whole Task 1 RT distribution, and not only for the fastest responses. Third, we reanalyzed our own data in Experiment $3 \mathrm{~b}$ after splitting Task 1 RTs into five bins. The corresponding ANOVA (including RT1 Bin as an additional factor) still revealed an effect of Task 2 trial type (indicating slower Task 1 responses on no-go Task 2 trials) and - most importantly-no significant interaction. ${ }^{3}$ In other words, the adverse no-go BCE was observed irrespective of the overall RT1 level.

The observation that an R1-R2 BCE was also present in the no-go trials of Experiments 1 and 2a suggests thatdespite the no-go status of the trial and the hypothesized reduction of task demands to a single Task 1 representation - some response-selection-related information regarding the stimuli's color dimension was processed nevertheless. Similar results were reported by Hommel (1998, Exp. 5; see also Janczyk, Renas et al., 2016, Exp. 2), in which participants were to give only R1 or R2 on each trial, according to a preceding cue. Even in this situation, the location of the signaled (but not executed) R2 affected Task 1 performance - thus, an R1-R2 BCE. It appears clear, then, that the mere perception of an $\mathrm{S} 2$ feature suffices to activate some response-related information. However, it is not clear which stage of Task 1 processing is affected. Although Hommel (1998) and Lien and Proctor (2002) suggested parallel stages of response activation as the source of (R1-R2) BCEs, other authors suggested that the capacitylimited stage of response selection is affected, instead (Janczyk et al., 2016; Thomson et al., 2015). Whether the $\mathrm{R} 1-\mathrm{R} 2$ and the no-go BCE emerge from the same or from different stages of processing cannot be decided on the basis of the present data. Although it is tempting to interpret the (nonsignificant) interactions in Experiments 1 and 2a in terms of the additive-factors logic (Sternberg, 1969), we prefer to be cautious here. First, the interactions were not significant on a conventional significance level, and second, some peculiarities of the results complicate a clear interpretation (see the Discussion section of Exp. 1). Thus, if anything, the pattern should be seen as additive, meaning that the two aspects may have affected different stages of processing.

\footnotetext{
${ }^{3}$ The main effect of RT1 bin was, of course, significant, $F(4,92)=74.05, p<$ $.001, \eta_{\mathrm{p}}{ }^{2}=.76, \varepsilon=.26$, with increasing RT1s $(396,472,534,615$, and $783 \mathrm{~ms}$, respectively). We further observed an almost significant main effect of Task 2 trial type, $F(1,23)=4.06, p=.056, \eta_{\mathrm{p}}{ }^{2}=.15$, with faster RT1s in Task 2 go trials $(546 \mathrm{~ms})$ than in Task 2 no-go trials $(574 \mathrm{~ms})$. Importantly, the interaction was not significant, $F(4,92)=0.43, p=.549, \eta_{\mathrm{p}}{ }^{2}=.02, \varepsilon=.30$.
} 


\section{Relation to other studies}

The adverse no-go BCE resembles results from a study by Huestegge and Koch (2014), which showed worse performance (there in terms of error rates, not RTs) in singleresponse versus dual-response conditions when the singleresponse condition (a keypress) involved the concurrent inhibition of a relatively automatic saccade. This paradigm did not involve a no-go stimulus, but just consisted of intermixed single- and dual-response trials triggered by the same aspect of a single stimulus (thus, this study was different from a typical dual-task setup). These results also highlight the role of automaticity: The more automatic a response is, the harder it is to suppress, eventually leading to greater cognitive costs of inhibition (doing nothing) than of overt execution (see also Raettig \& Huestegge, 2016). As in the present study, automaticity was assumed to increase the tendency to translate a stimulus into a response (similar to advance preparedness, based on the fact that a no-go stimulus is presented second or that Task 2 is a simple go/no-go task with only one response alternative).

The inhibition of prepared (and already executed) responses is also investigated through stop-signal experiments (Lappin \& Eriksen, 1966; Logan, Cowan, \& Davis, 1984). In such experiments, participants are presented a go stimulus signaling a particular response on the majority of trials. Unpredictably, on some trials a second stimulus (the "stop signal") is presented after a variable delay, which signals to immediately interrupt and stop the already-ongoing response processing. This active interruption also has consequences for other ongoing responses (see Aron, 2011). However, it should be noted that this situation is very different from a no-go paradigm (see also Verbruggen \& Logan, 2008), in which participants are never explicitly instructed to start response processing prior to a subsequent cancellation signal.

\section{Conclusion}

Whether a Task 2 no-go stimulus affects Task 1 performance in a dual-task setting beneficially or adversely appears to depend on whether or not particular features of a response (or the whole response) are likely to be prepared in advance. If so, encountering a no-go stimulus hampers Task 1 performance, likely via inhibitory demands. Otherwise, Task 1 performance is much improved, most likely because participants can utilize the no-go information to reduce the task demands to a single - task. More specifically, our overall results suggest that inhibitory costs in dual-tasking can be avoided by establishing a situation in which (a) inhibitory information is provided early and (b) the to-beinhibited task demands are sufficiently complex to reduce the likelihood of advance (response) preparation.
Author note The work of M.J. is supported by the Institutional Strategy of the University of Tübingen (Deutsche Forschungsgemeinschaft/German Research Foundation; ZUK 63). Furthermore, this work was supported by a grant from the Ministry of Science, Research and the Arts of the state of Baden-Württemberg. We thank Bernhard Hommel and one anonymous reviewer for helpful comments on a previous version of the manuscript.

\section{References}

Aron, A. R. (2011). From reactive to proactive and selective control: Developing a richer model for stopping inappropriate responses. Biological Psychiatry, 69, e55-e68. doi:10.1016/j. biopsych.2010.07.024

Berlyne, D. E. (1957). Conflict and choice time. British Journal of Psychology, 48, 106-118.

Ellenbogen, R., \& Meiran, N. (2008). Working memory involvement in dual-task performance: Evidence from the backward compatibility effect. Memory \& Cognition, 36, 968-978. doi:10.3758 /MC.36.5.968

Hommel, B. (1998). Automatic stimulus-response translation in dualtask performance. Journal of Experimental Psychology: Human Perception and Performance, 24, 1368-1384. doi:10.1037/00961523.24.5.1368

Hommel, B., \& Eglau, B. (2002). Control of stimulus-response translation in dual-task performance. Psychological Research, 66, 260273.

Huestegge, L., \& Koch, I. (2014). When two actions are easier than one: How inhibitory control demands affect response processing. Acta Psychologica, 151, 230-236.

Janczyk, M. (2016). Sequential modulation of backward crosstalk and task-shielding in dual-tasking. Journal of Experimental Psychology: Human Perception and Performance, 42, 631-647.

Janczyk, M., Büschelberger, J., \& Herbort, O. (2016). Larger betweentask crosstalk in children than in adults: Behavioral results from the backward-crosstalk paradigm and a diffusion model analysis. Journal of Experimental Child Psychology.

Janczyk, M., Nolden, S., \& Jolicœur, P. (2015). No differences in dualtask costs between forced- and free-choice tasks. Psychological Research, 79, 463-477.

Janczyk, M., Pfister, R., Hommel, B., \& Kunde, W. (2014). Who is talking in backward crosstalk? Disentangling response- from goalconflict in dual-task performance. Cognition, 132, 30-43. doi:10.1016/j.cognition.2014.03.001

Janczyk, M., Pfister, R., Wallmeier, G., \& Kunde, W. (2014). Exceptions from the PRP effect? A comparison of prepared and unconditioned reflexes. Journal of Experimental Psychology: Learning, Memory, and Cognition, 40, 776-786.

Janczyk, M., Renas, S., \& Durst, M. (2016). Identifying the locus of compatibility-based backward crosstalk: Evidence from an extended PRP paradigm. Manuscript submitted for publication.

Ko, Y.-R., \& Miller, J. (2014). Locus of backward crosstalk effects on Task 1 in a psychological refractory period task. Experimental Psychology, 61, 30-37.

Lappin, J. S., \& Eriksen, C. W. (1966). Use of a delayed signal to stop a visual reaction-time response. Journal of Experimental Psychology, $72,805-811$.

Lien, M.-C., \& Proctor, R. W. (2000). Multiple spatial correspondence effects on dual-task performance. Journal of Experimental Psychology: Human Perception and Performance, 26, 1260-1280.

Lien, M.-C., \& Proctor, R. W. (2002). Stimulus-response compatibility and psychological refractory period effects: Implications for response selection. Psychonomic Bulletin \& Review, 9, 212-238. doi:10.3758/BF03196277 
Logan, G. D., Cowan, W. B., \& Davis, K. A. (1984). On the ability to inhibit simple and choice reaction time responses: A model and a method. Journal of Experimental Psychology: Human Perception and Performance, 10, 276-291. doi:10.1037/0096-1523.10.2.276

Miller, J. (2006). Backward crosstalk effects in psychological refractory period paradigms: Effects of second-task response type on first-task response latencies. Psychological Research, 70, 484-493.

Miller, J., \& Durst, M. (2014). "Just do it when you get a chance": The effects of a background task on primary task performance. Attention, Perception \& Psychophysics, 76, 2560-2574.

Miller, J., \& Durst, M. (2015). A comparison of the psychological refractory period and prioritized processing paradigms: Can the responseselection bottleneck model explain them both? Journal of Experimental Psychology: Human Perception and Performance, 41, 1420-1441.

Mittelstädt, V., \& Miller, J. (2016). Separating limits on preparation versus on-line processing in multitasking paradigms: Evidence for resource models. Journal of Experimental Psychology: Human Perception and Performance.

Pashler, H. (1994). Dual-task interference in simple tasks: Data and theory. Psychological Bulletin, 116, 220-244. doi:10.1037/00332909.116.2.220
Raettig, T., \& Huestegge, L. (2016). The hard work of doing nothing: Accounting for inhibitory costs during multiple action control. Manuscript submitted for publication.

Röttger, E., \& Haider, H. (2016). Investigating the characteristics of "not responding": Backward crosstalk in the PRP paradigm with forced vs. free no-go decisions. Psychological Research.

Sternberg, S. (1969). The discovery of processing stages: Extension of Donders' method. Acta Psychologica, 30, 276-315. doi:10.1016 /0001-6918(69)90055-9

Telford, C. W. (1931). The refractory phase of voluntary and associative responses. Journal of Experimental Psychology, 14, 1-36. doi: $10.1037 / \mathrm{h} 0073262$

Thomson, S. J., Danis, L. K., \& Watter, S. (2015). PRP training shows Task 1 response selection is the locus of the backward response compatibility effect. Psychonomic Bulletin \& Review, 22, 212-218.

Verbruggen, F., \& Logan, G. D. (2008). Automatic and controlled response inhibition: Associative learning in the go/no-go and stopsignal paradigms. Journal of Experimental Psychology: General, 137, 649-672. doi:10.1037/a0013170

Welford, A. T. (1952). The "psychological refractory period" and the timing of high-speed performance - A review and a theory. British Journal of Psychology, 43, 2-19. 